\title{
Réapprendre à apprendre
}

\section{Monsieur,}

Étudiant en médecine dans les années 80, j'admets n'avoir pas éétellement ébloui par le modèe pédagogique alors en vigueur. Issu des mathématiques, je m'ennuyais passablement, comprenant mal comment cette masse de données brutes fini rait un jour par avoir un sens. Allaisje réussir à me donner, à partir de concepts aussi disparates qu'indigetes, unelogi queou à tout lemoinsuneapprochemédicale cohérente? Jen'en savais rien.

La pensée médicale métait alors s peu évidente que je me demandais bien pourquoi on exigeait une formation en sciences pures pour accéder à ce cénade Lire le bottin téé phonique, exerciceautrement stimulant pour l'hippocampe mais un peu snistre pour le cortex, me faisait à peu prè le même effet. Bien vite, songeant queje n'étais pas tout à fait à ma place, j'étais plus souvent au journal étudiant qu'en classe à partir de la seconde année. $M$ aiş, après un détour prolongépar l'Afrique, j'ai finalement réal isé, à l'hôpital, la nature absolument fascinante d'un métier qui n'allait jamais plus me décevoir.

Puis, l'an dernier, grâce à la suggetion d'un collègue cardiologue, j'ai vécu la curieuse expérience de retourner à ma chère faculté, histoire d'y enseigner le cours de cardiologie aux étudiants de $2^{\circ}$ année $D$ ans mon groupe de tutorat, neuf étudiants, auss stimulésquestimulants, paraissant fascinés par cequ'ilsapprenaient, en redemandaient jour après jour. Réfléchir, approfondir, rechercher de nouvellespistes et explorer semblait être, pour eux, auss naturel qu'avait éé, pour moi, à l'époque, l'écriture de mes di atri bes pour le journal. La médecine, univers à défricher et à comprendre plutôt que gavage d'oies? Était-ce possible?

Bien entendu, la différence était dansla manière et non dansla matière. D epui squequesannées, grâceà la persévé rance d'esprits audacieux, la faculté avait intégrél'apprenti ssage par problème (APP) dans sa pédagogie: analyseet résolution plutôt qu'archivage passif de la sixième transormation enzymatique du cycle de Krebs. U ne approche au fond si semblableà la penséeclinique, et donc menant directement à celleci. J'étais comblé

Le hasard faisant bien les choses, j'ai vécu, quelques mois plus tard, ce qui a été, malgré 700 heures d'E M C (Enseignement M édical Continu) accumulées, sûrement le congrès le plus remarquable de ma carrière. Je n'ai pas dit « assister »; j'aurais pu choisir « participer » mais « vivre» est ici le plus juste terme, ayant moi-même contribué, comme chacun des participants, à la réussite de cette semaine de formation, réussi te à la mesure de l'énergie investie

Un solide cours de médecine (d'urgence) preuves à l'appui (M PA*), élaboré par une équipe de l'urgence de l'H ôpital Général Juif, en collaboration avec l'Association des méde cins d'urgence du Q uébec. Calquée sur celle donnée chaque annéeà l'U niversité M cM aster, la formation fut étirée sur une pleine semaine, dix heures par jour, plus des heures nombreuses de préparation, l'expérience fut auss captivante qu'épui sante. À la fin, nousétionséreintés, mais heureux, et surtout intellectuellement comblés.

Composé essentiellement d'ate iers interactifs, très vivants, où, tour à tour, nousallions êreanimateurs, évaluateursou participants, le cours visait à nous permettre d'approfondir et de mieux comprendre les principes fondamentaux de I'analysemodernedela littératuremédicale. Chaqueatelier, portant sur un artide récent, illustrait ainsi une catégorie spécifique: investigation, thérapeutique, guide de pratique, revue systématique, etc. N ous avons appliqué, pour chacune, la méthode la plus appropriée de révision critique, accompagnés dans ce cheminement par deux médecinsexperts. D escollèguesayant montédèslesecond jour unescé nographie auss cohérente que parfaitement comique, nous avons dès lors plenement embarqué dans desjeux de rôles

*M PA (M édecine Preuves à l'A ppui) est un acronyme utilisé au Q uébec pour traduire EBM (Evidence Based M edecine). 


\section{Lettres à l'éditeur}

délirants, qui rendaient I'apprentissage aussi ludique qu'efficace; jusqu'à la toutefin, leséquipesont ains rivalisé lesunesavec lesautres, dansun esprit d'émulation vivifiant. Pour dire vrai, je n'y ai pas appris de nouvelles notions, ni derecette. Pourtant, j'ai apprislà plusquejamais: à l'image du renouvellement du cours de médecine, j'ai réappris à apprendre, en quelque sorte; je me suis outillé pour mieux appréhender la médecine elle-même, ses fondements, son évolution et surtout sa pratique, chaque atelier visant, en effet, à répondre à une question clinique précise, de la manièrela plus rigoureusequi soit.

Les courbes d'apprentissage font souvent, de ces premières expériences, une telle aventure grisante. Cette semaine de M PA n'a pasfait pas exception, peut-être un peu parce que j'ai compristant de choses en si peu de jours, moi qui, sans être chevronné en M PA, ne me considère pas non plus comme un illettré médical. M ais je réalise à quel point ce mode de formation, si efficace, est encore à peu près absent denotreEM C, où nous préférerons lesapprentissages passifs, accumulation de notions et de recettes, parfois servies sans I'approfondi ssement requis. Pourtant, nous manquons encore trop souvent de sens critique quand il est question, par exemple, denouveautés médicales.

II est urgent que notre EM C soriente vers ce type d'appren- tissage interactif, en petits ateliers, visant à faciliter la compréhension et l'analyse plutôt que la transmission du contenu. Ce que nous perdrons en exhaustivité, nous le gagnerons en profondeur et en rigueur, vertus d'autant plus fondamentales que les outils d'accès à la connai ssance sont aujourd'hui très largement disponibles, rendant caduque la nécessité d'accumuler, sans discernement, des notions hétérodites.

Si nous ne prenons pas ce virage, si nous ne consacrons pas toutes nosénergi es et une bonne part de notre $\mathrm{EM} C$ à déve lopper detelleshabiletés, il nefait pas dedoutequela science ne sera bientôt plus, pour la majorité d'entre nous, qu'une sorte devernis, rudement misà l'épreuvedansun monde où lestechniques de persuasion dépassent largement notrecapacitéderésistance. En continuitéavec lemonde del'enseignement universitaire, il nous faut donc travailler ensembleà cette révolution : cessons d'apprendre, réapprenons à apprendre

C'est un gage de survie pour l'indépendance et l'avenir de notreprofession.

Alain Vadeboncoeur M D. U rgentologue, Institut de cardiologie de M ontréal Ce texte, déjà publié dans M edActuel FM C, juillet-août 2002, est reproduit avec autorisation de la rédaction de cette revue.

\section{www.pedagogie-medicale.org \\ un site à votre disposition}

Votre revue est disponible sur Internet. Vous y retrouverez les informations général es sur la revue, les adresses de contact, les sommaires des différents numéros parus, les directives aux auteurs etc. Les éditoriaux et toutes les rubriques de la section «Vie pédagogique » y sont disponibles « in extenso » en format pdf (lisibles avec Acrobat Reader). A u fur et à mesure de leur publication, lesfiches pratiques seront mises en ligne sur lesite. Vous pourrez ainsi les télécharger, les imprimer chez vous, et vous construire progressivement votre propremanuel pratique. D ivers liens vers les auteurs des articles déjà publiés et les organisations du comité de parrainage sont égal ement disponibles.

\section{Enfin, vous pourrez accéder aux forums de discussion, et nous faire part par ce canal de vos commen- taires, vos critiques ou vos attentes. Une nouvelle rubrique a été ouverte début mars 2002 : « Vos réac- tions aux articles publiés ». N'hésitez pas à nous transmettre vos opinions. Nous les ferons suivre aux auteurs, qui le cas échéant y répondront. Les discussions les plus intéressantes seront publiées dans les pages de la revue.}

Le site www.pedagogie-medicale.org se veut un lien amical et fonctionnel entre tous ceux qui sont impliqués dans la formation médicale. Le site doit leur permettre de partager leurs préoccupations et leurs solutions dans leur action pour la formation initiale et la formation continue de nos professionnels de santé.

La rédaction 